\title{
Erratum to: Enhanced serine production by bone metastatic breast cancer cells stimulates osteoclastogenesis
}

\author{
Sirkku Pollari · Sanna-Maria Käkönen · Henrik Edgren • \\ Maija Wolf · Pekka Kohonen · Henri Sara • \\ Theresa Guise $\cdot$ Matthias Nees $\cdot$ Olli Kallioniemi
}

Published online: 13 July 2011

(C) Springer Science+Business Media, LLC. 2011

\section{Erratum to: Breast Cancer Res Treat \\ DOI 10.1007/s10549-010-0848-5}

The authors would like to inform that the description for the data sets used in the Kaplan-Meier analysis of relapsefree survival and the number of samples used in the Kaplan-Meier analysis of overall survival were incorrectly reported in the original publication. PSAT1 expression was analyzed in almost twice as many samples as reported. The results and significance of all the analyses remain unchanged. It should read as follows:

The online version of the original article can be found under doi: 10.1007/s10549-010-0848-5.

S. Pollari $(\square) \cdot$ P. Kohonen $\cdot$ H. Sara $\cdot$ M. Nees

Medical Biotechnology, VTT Technical Research Center

of Finland and Turku Center for Biotechnology,

Turku, Finland

e-mail: ext-sirkku.pollari@vtt.fi

\section{S.-M. Käkönen}

Institute of Biomedicine, Department of Anatomy,

University of Turku, Turku, Finland

H. Edgren · M. Wolf · O. Kallioniemi

Institute for Molecular Medicine Finland (FIMM),

University of Helsinki, Helsinki, Finland

T. Guise

Division of Endocrinology, Indiana University,

Indianapolis, IN, USA

Present Address:

S.-M. Käkönen

Amgen AB Finland, Espoo, Finland

\section{Materials and methods}

Association of PHGDH, PSAT1, PSPH, and SLC1A4 expression with time to relapse in the Affymetrix gene expression studies GSE6532, GSE12276, and GSE9195 (samples analyzed with U133 Plus 2.0 Array, $n=368$ ) and with overall survival in the Affymetrix gene expression studies GSE3494 and GSE1456 $(\boldsymbol{n}=\mathbf{3 8 2}$ for PHGDH, PSPH, SLC1A4; $\boldsymbol{n}=\mathbf{7 6 4}$ for PSAT1) was assessed by Kaplan-Meier analysis.

\section{Results}

First, we analyzed the correlation of PHGDH, PSAT1, PSPH, and SLC1A4 expression to time to relapse in 368 breast cancers and to overall survival time in $\mathbf{3 8 2}$ (PHGDH, PSPH, SLC1A4) or 764 breast cancers (PSAT1).

The Fig. $6 \mathrm{~b}$ with the corrected sample numbers is given below. 

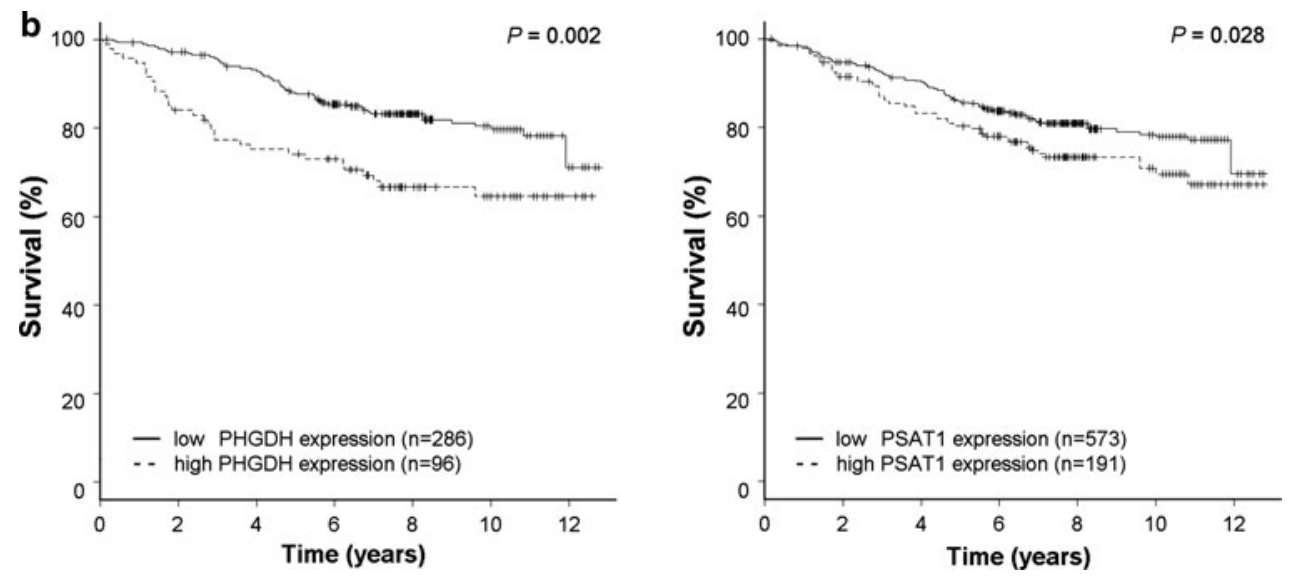